EPJ Web of Conferences 112, 03003 (2016)

DOI: $10.1051 /$ epjconf/201611203003

(C) Owned by the authors, published by EDP Sciences, 2016

\title{
Progress on nuclear modifications of structure functions
}

\author{
S. Kumano ${ }^{1,2}$ \\ ${ }^{1}$ KEK Theory Center, Institute of Particle and Nuclear Studies, KEK, \\ 1-1, Ooho, Tsukuba, Ibaraki, 305-0801, Japan \\ ${ }^{2}$ J-PARC Branch, KEK Theory Center, Institute of Particle and Nuclear Studies, KEK, \\ and Theory Group, Particle and Nuclear Physics Division, J-PARC Center, \\ 203-1, Shirakata, Tokai, Ibaraki, 319-1106, Japan
}

\begin{abstract}
We report progress on nuclear structure functions, especially on their nuclear modifications and a new tensor structure function for the deuteron. To understand nuclear structure functions is an important step toward describing nuclei and QCD matters from low to high densities and from low to high energies in terms of fundamental quark and gluon degrees of freedom beyond conventional hadron and nuclear physics. It is also practically important for understanding new phenomena in high-energy heavy-ion collisions at RHIC and LHC. Furthermore, since systematic errors of current neutrinooscillation experiments are dominated by uncertainties of neutrino-nucleus interactions, such studies are valuable for finding new physics beyond current framework. Next, a new tensor-polarized structure function $b_{1}$ is discussed for the deuteron. There was a measurement by HERMES; however, its data are inconsistent with the conventional convolution estimate based on the standard deuteron model with D-state admixture. This fact suggests that a new hadronic phenomenon should exist in the tensor-polarized deuteron at high energies, and it will be experimentally investigated at JLab from the end of 2010's.
\end{abstract}

\section{Introduction}

Nuclear structure functions are different from corresponding ones of the nucleon. Such nuclear modifications have been measured from relatively small $x(\sim 0.005)$ to large $x(\sim 0.7)$ mainly in chargedlepton deep inelastic scattering (DIS). Physics mechanisms for the nuclear effects are different in each $x$ region [1]. At small $x$, a virtual photon becomes $q \bar{q}$ (or vector mesons), which interacts with a nucleus by strong interactions. It leads to nuclear shadowing phenomena due to multiple interactions in a nucleus. At medium $x$, there are negative contributions from nuclear binding and possible internal nucleon modifications. The modifications are positive at large $x$ due to nucleon's Fermi motion in a nucleus. Although the major nuclear-modification mechanisms are known, we cannot calculate the structure functions precisely, typically less than $10 \%$ accuracy, by the theoretical models. For actual application to heavy-ion physics and neutrino reactions, it is practically not appropriate to reply on such models. For example, neutrino-nucleus cross sections need to be calculated within 5\% accuracy in future leptonic CP violation measurements [2]. Therefore, it is desirable to use global analysis results as a model for the nuclear parton distribution functions (NPDFs) [3, 4], in the same way as the global analysis PDFs are used for calculating cross sections at LHC to find physics beyond the standard model. 
The NPDFs are determined by analyzing world data on high-energy nuclear reactions, including charged-lepton DIS, neutrino DIS, Drell-Yan, and hadron productions. Currently, there are two major issues in the NPDF studies. One is that gluon shadowing is not determined from the current measurements $[3,4]$, and the other is that modifications suggested by the neutrino DIS data could be different from the ones which are inferred from the charged-lepton DIS [5]. On the first point, the issue is due partly to the fact that a lepton-nucleus collider similar to HERA does not exist to observe scaling violation at small $x$. However, the situation could improve because LHC started producing data, which are sensitive to small- $x$ physics. On the second point, somewhat conflicting results are obtained among different analysis groups. It is desirable to clarify the situation by another serious analysis to discuss the details of analysis conditions and data handling. In this report, we discuss the situation of these studies in Sec. 2.

As the second topic, we explain tensor structure functions of the deuteron in charged-lepton DIS with a polarized deuteron [6]. The deuteron structure is well known at low energies and it is described by a proton-neutron $\mathrm{S}$-wave bound state with small $\mathrm{D}$-wave admixture. Since the deuteron is a spin-1 hadron, it has new polarized structure functions, $b_{1}, b_{2}, b_{3}$, and $b_{4}$ in addition to the four structure functions, $F_{1}, F_{2}, g_{1}$, and $g_{2}$, which exist for the spin-1/2 nucleon in the charged-lepton DIS. The structure functions $b_{1}$ and $b_{2}$ are leading-twist ones, which are related by the Callan-Gross like relation $2 x b_{1}=b_{2}$ in the scaling limit. The $b_{3}$ and $b_{4}$ are higher-twist ones.

There are only a few $b_{1}$ data measured by the HERMES collaboration [7]. Although much accurate measurements are needed, there is already an interesting hint in the data toward a new discovery. The most conventional way to estimate $b_{1}$ theoretically is to use a convolution model, where $b_{1}$ is calculated by an unpolarized structure function of the nucleon with nucleon-momentum distributions including the D wave. The HERMES data are an order-of-magnitude larger than this standard model predication. It indicates that $b_{1}$ cannot be understood by the conventional model, and a new hadronic physics should be introduced for interpretation of the HERMES data. Fortunately, an experimental proposal to JLab was approved for $b_{1}$, and its measurement will start around the year of 2019 [8]. It is a good opportunity to find a new exotic hadronic phenomenon in the simplest nucleus, deuteron. The deuteron tensor structure used to play an important role in low-energy nuclear physics. It is now time to understand the tensor structure in terms of quark and gluon degrees of freedom with new hadron physics. We explain this current situation as the second topic in Sec. 3.

\section{Nuclear parton distribution functions}

Nuclear parton distributions are modified from the ones for the nucleon. The space is limited for this article, so that we do not address ourselves to physics mechanisms. An interested reader may read summary articles in Ref. [1]. We focus our discussions on the status of NPDFs and an application to neutrino physics. The NPDFs are determined by a global analysis of world data on high-energy lepton-nucleus, proton-nucleus, and nucleus-nucleus reactions. The distributions are parametrized, and optimum parameters are determined by a $\chi^{2}$ analysis. There are a few groups which investigate the NPDFs. There are two types for the parametrization. One is to obtain modifications from typical nucleonic PDFs, and the other is to obtain the NPDFs directly. The latter one is a general way of analysis on the same footing with the nucleonic PDF analysis, whereas it is easier to impose physical constrains in the former one because the determined NPDFs could become unphysical distributions due to the lack of data especially at extreme kinematical conditions. If we were to have sufficient experimental data, both results should agree with each other.

There are two major issues in the determination of NPDFs. First, nuclear modifications of the gluon distribution cannot be fixed due to the lack of data which are sensitive to them. Second, some 
inconsistencies were pointed out for the modifications between charged-lepton and neutrino scattering processes [5], although some other analysis do not show such inconsistency clearly. In the following, we first explain a connection to neutrino physics and it is partially related to the second issue.

Precise NPDFs are needed for finding any new physics in high-energy nuclear reactions such as properties of quark-gluon matters in heavy-ion collisions at RHIC and LHC. There is another important application to neutrino physics. The statistical errors in neutrino-oscillation measurements become smaller and smaller, but their systematic errors are still dominated by neutrino-nucleus interactions, which is an obstacle for a new discovery. There are three major kinematical regions as shown in Fig. 1: quasi-elastic (QE), resonance (RES), and deep inelastic scattering (DIS). There are not definite boundaries in the sense that $W^{2}$ and $Q^{2}$ cut values depend on researchers. However, a usual choice is the cut $W^{2} \geq 4 \mathrm{GeV}^{2}$ for the DIS, and $Q^{2}$ should be large enough, typically $Q^{2} \geq 1$ $\mathrm{GeV}^{2}$. These three regions have been investigated by dif-

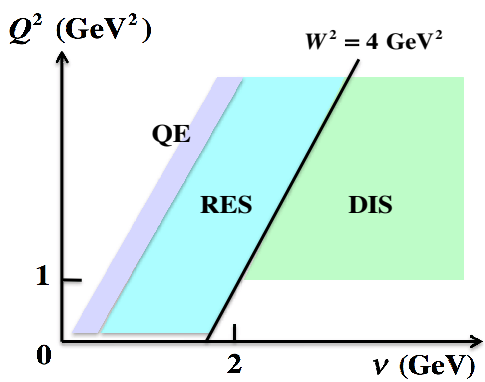

Figure 1. Kinematical regions of neutrinonucleus scattering. Here, QE, RES, and DIS indicate quasi-elastic, resonance, and deep inelastic scattering.

ferent types of physicists. In the current neutrino experiments, except for the ultra-high energy IceCube experiment, typical energies are from several hundred $\mathrm{MeV}$ to a few dozen $\mathrm{GeV}$. Therefore, all of these kinematical cross sections should be precisely known for reducing the systematical uncertainties in neutrino oscillation measurements. For future leptonic $\mathrm{CP}$ violation measurements, the cross sections need to be understood within the 5\% level. A project is in progress to provide a code to calculate the cross section in any kinematical range by combining theories of different regions at the J-PARC branch of the KEK theory center [2]. In the following, we discuss only the DIS studies and such a unification effort will be explained elsewhere.

If there were no nuclear modification, the NPDFs are simple addition of proton and neutron contributions. However, from experimental measurements, nuclear medium effects are typically 10-20\% depending on a nuclear size. Such medium effects are parametrized by the function $w_{i}$ in Ref. [3] as

$$
f_{i}^{A}\left(x, Q_{0}^{2}\right)=w_{i}(x, A, Z) \frac{1}{A}\left[Z f_{i}^{p}\left(x, Q_{0}^{2}\right)+N f_{i}^{n}\left(x, Q_{0}^{2}\right)\right],
$$

where $p$ and $n$ indicate the proton and neutron, $A, Z$, and $N$ are mass number, atomic number, and neutron number, $i$ indicates a type of distribution $\left(i=u_{v}, d_{v}, \bar{q}, g\right)$, and $Q_{0}^{2}$ is the initial $Q^{2}$ scale. The functions $w_{i}$ are expressed by a number of parameters, which are then determined by a global analysis. The kinematical range of $x$ is $0<x<A$ for a nucleus. Therefore, the functional form of Eq. (1) cannot describe the region $x>1$. However, there is no DIS data in such a large- $x$ region, so it is not a serious problem at this stage. In the HKN analysis [3], the nuclear modification functions are expressed in terms of the parameters, $\alpha, \beta, a_{i}, b_{i}, c_{i}$, and $d_{i}$ as

$$
w_{i}(x, A, Z)=1+\left(1-\frac{1}{A^{\alpha}}\right) \frac{a_{i}+b_{i} x+c_{i} x^{2}+d_{i} x^{3}}{(1-x)^{\beta}},
$$

at $Q_{0}^{2}=1 \mathrm{GeV}^{2}$. The parameters are determined by a global $\chi^{2}$ analysis with the standard DGLAP evolution equation in comparison with the world data. 
As an example, nuclear modifications of ${ }^{40} \mathrm{Ca}$ PDFs and their uncertainties are shown for the next-to-leading (NLO) order HKN parametrization [3] in Fig. 2 at $Q^{2}=1 \mathrm{GeV}^{2}$. There are some differences in the NPDFs from other groups [4] at large $x$ especially for the antiquark and gluon distributions because there are few data which constrain them. In addition, large differences exist in the gluon distributions at small $x$. The nucleonic gluon distribution is constrained by the scaling violation of $F_{2}$ from the HERA ep-collider experiments, whereas there is no such a measurement for nuclei at small $x$. However, the situation may improve in the near future

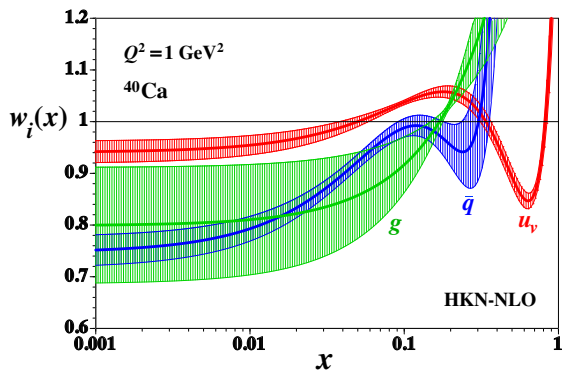

Figure 2. HKN nuclear modifications for ${ }^{40} \mathrm{Ca}$ at $Q^{2}=1 \mathrm{GeV}^{2}$. due to LHC measurements with heavy ions. Using the obtained NPDFs together with DGLAP evolution equations, one can calculate nuclear modifications of structure functions in neutrino scattering. Some inconsistencies were reported between the charged-lepton DIS and neutrino DIS structure functions in Ref. [5]; however, the differences are not clear in other groups [4]. Our analysis is now in progress by including neutrino data for clarifying this issue as an independent trial.

\section{New spin structure function $b_{1}$ for deuteron}

There are four new structure functions, $b_{1}, b_{2}, b_{3}$, and $b_{4}$, for the spin-1 hadron in charged-lepton deep inelastic scattering. They are defined in the hadron tensor as [6]:

$$
\begin{aligned}
W_{\mu \nu}^{\lambda_{f} \lambda_{i}}= & -F_{1} \hat{g}_{\mu \nu}+\frac{F_{2}}{M v} \hat{p}_{\mu} \hat{p}_{\nu}+\frac{i g_{1}}{v} \epsilon_{\mu \nu \lambda \sigma} q^{\lambda} s^{\sigma}+\frac{i g_{2}}{M v^{2}} \epsilon_{\mu \nu \lambda \sigma} q^{\lambda}\left(p \cdot q s^{\sigma}-s \cdot q p^{\sigma}\right) \\
& -b_{1} r_{\mu \nu}+\frac{1}{6} b_{2}\left(s_{\mu \nu}+t_{\mu \nu}+u_{\mu \nu}\right)+\frac{1}{2} b_{3}\left(s_{\mu \nu}-u_{\mu \nu}\right)+\frac{1}{2} b_{4}\left(s_{\mu \nu}-t_{\mu \nu}\right),
\end{aligned}
$$

where $p$ and $q$ are spin- 1 hadron and virtual-photon momenta, $v$ is the energy transfer, and $r_{\mu \nu}, s_{\mu \nu}, t_{\mu \nu}$, and $u_{\mu \nu}$ are defined by the polarization vector of the spin-one hadron $E^{\mu}$ in Ref. [6]. The spin vector $s$ is expressed by the polarization vector as $\left(s_{\lambda_{f} \lambda_{i}}\right)^{\mu}=-i \epsilon^{\mu \alpha \alpha \beta} E_{v}^{*}\left(\lambda_{f}\right) E_{\alpha}\left(\lambda_{i}\right) p_{\beta} / M$ where the initial and final polarization vectors are denoted as $E^{\mu}\left(\lambda_{i}\right)$ and $E^{\mu}\left(\lambda_{f}\right)$ with the spin states $\lambda_{i}$ and $\lambda_{f}$, and $M$ is the mass of the spin-1 hadron. The coefficients of $b_{1}, b_{2}, b_{3}$ and $b_{4}$ are defined as symmetric under $\mu \leftrightarrow v$ in Eq. (3), and they vanish under the spin average. They are also defined so that $b_{1}$ and $b_{2}$ are twisttwo functions to satisfy the Callan-Gross type relation, $2 x b_{1}=b_{2}$. The $b_{3}$ and $b_{4}$ are higher-twist structure functions. The twist-two structure function $b_{1}$ is expressed in terms of the tensor-polarized parton distributions $\delta_{T} q$ and $\delta_{T} \bar{q}$ as

$$
b_{1}\left(x, Q^{2}\right)=\frac{1}{2} \sum_{i} e_{i}^{2}\left[\delta_{T} q_{i}\left(x, Q^{2}\right)+\delta_{T} \bar{q}_{i}\left(x, Q^{2}\right)\right], \quad \delta_{T} q_{i} \equiv q_{i}^{0}-\frac{q_{i}^{+1}+q_{i}^{-1}}{2} .
$$

Here, $i$ indicates the flavor of a quark, $e_{i}$ is a quark charge, and $q_{i}^{\lambda}$ indicates an unpolarized-quark distribution in the hadron spin state $\lambda$. The tensor-polarized distribution is much different from the longitudinally-polarized one $\Delta q=q_{\uparrow}-q_{\downarrow}$, where $\uparrow$ and $\downarrow$ indicate the polarization of a quark along the hadron-spin direction, in the sense that it is "unpolarized"-quark distribution in a tensor-polarized spin-1 hadron.

The structure function $b_{1}$ can be calculated by the standard convolution picture for the deuteron in terms of parton-momentum distributions convoluted with nucleon momentum distributions. The $b_{1}$ is associated with tensor structure of the deuteron, so that $\mathrm{D}$-wave admixture should be properly considered for the convolution estimation. However, it is surprising to find an order-of-magnitude 
difference between such a standard-model estimate and experimental data obtained by the HERMES collaboration, although the data have large errors. There are other theoretical-model calculations; however, we do not step into such theoretical models in this article, and we discuss probable tensorpolarized distributions from the HERMES data.

Such a parametrization was studied in Ref. [9]. We consider the following constraint in the form of $b_{1}$ sum rule [10], which was obtained in a parton model and it is similar to the Gottfried sum:

$$
\begin{aligned}
\int d x b_{1}(x) & =0+\frac{1}{9} \int d x\left[4 \delta_{T} \bar{u}(x)+\delta_{T} \bar{d}(x)+\delta_{T} \bar{s}(x)\right], \\
\int \frac{d x}{x}\left[F_{2}^{p}(x)-F_{2}^{n}(x)\right] & =\frac{1}{3}+\frac{2}{3} \int d x[\bar{u}(x)-\bar{d}(x)] .
\end{aligned}
$$

As the Gottfried-sum-rule violation created a field of flavor-symmetric light-antiquark distributions $(\bar{u} \neq \bar{d})[11]$, a finite $b_{1}$ sum could indicate a finite tensor-polarized antiquark distribution, which cannot be expected from ordinary theoretical ideas. For the time being as the first-step study, we neglect such exotic contributions and consider the condition $\int d x b_{1}(x)=0$ for constraining the tensor distributions.

As the first trial, we consider the case that a certain fraction $\left(\delta_{T} w\right)$ of unpolarized PDFs is tensor polarized in the deuteron [9]: $\delta_{T} q_{i v}^{D}(x)=\delta_{T} w(x) q_{i v}^{D}(x), \delta_{T} \bar{q}_{i}^{D}(x)=\alpha_{\bar{q}} \delta_{T} w(x) \bar{q}_{i}^{D}(x)$ for the tensorpolarized parton distributions. Here, we assume a common function $\delta_{T} w(x)$ for both quarks and antiquarks except for a different overall constant $\alpha_{\bar{q}}$. Now, the problem becomes how to determine $\delta_{T} w(x)$. We assume that the valence-quark distributions should satisfy the sum $\int d x\left(b_{1}\right)_{\text {valence }}=0$. Then, the function $\delta_{T} w(x)$ should have a node at least, so we may take the parametrization $\delta_{T} w(x)=$ $a x^{b}(1-x)^{c}\left(x_{0}-x\right)$. Here, $a, b$, and $c$ are the parameters determined by the analysis, and the node position $x_{0}$ is expressed by the other parameters as $x_{0}=\int d x x^{b+1}(1-x)^{c}\left(u_{v}+d_{v}\right) / \int d x x^{b}(1-x)^{c}\left(u_{v}+\right.$ $d_{v}$ ) due to the sum rule. Such a node also exists in $b_{1}$ calculated by the convolution model with the D-state admixture.

We tried two scenarios depending on whether or not the antiquark tensor polarization exists:

- Set 1: Tensor-polarized antiquark distributions are terminated $\left(\alpha_{\bar{q}}=0\right)$.

- Set 2: Finite tensor-polarized antiquark distributions are allowed ( $\alpha_{\bar{q}}$ is a parameter).

The parameters are determined by a $\chi^{2}$ analysis in each case. Determined $b_{1}$ and tensor-polarized PDFs are shown in Figs. 3 and 4. There are two curves in Fig. 3 for the sets 1 and 2. It is obvious from this figure, within the restriction of the used parametrization, the HERMES data cannot be explained without the antiquark tensor polarization. Namely, $\chi^{2}$ is much larger for the set- 1 analysis. The obtained tensor distributions are shown in Fig. 4. The dashed curve indicates the valence-quark distribution in the set 1 . The solid and dotted curves indicate the valence-quark and antiquark distributions in the set 2 . The valence-quark distribution is negative at large $x(>0.3)$ and then it turns into

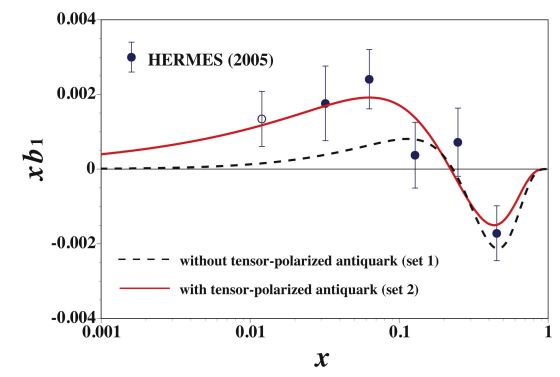

Figure 3. Obtained $b_{1}$ and HERMES data [9].

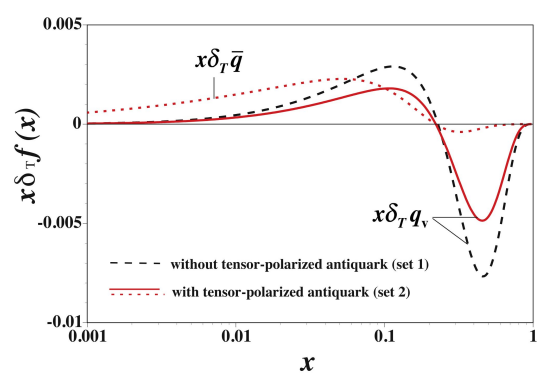

Figure 4. Determined tensor-polarized distributions [9]. 
positive at $x \simeq 0.2$. The antiquark distributions are especially large at small $x(<0.1)$. Since there are only a few data points at this stage, there are large uncertainties for the distributions.

In future, we need theoretical and experimental efforts on $b_{1}$ physics. On the theory side, we need to understand the obtained tensor-polarized distributions in Fig. 4 by appropriate theoretical models. The standard deuteron model with the D-state admixture cannot explain the distributions because a typical convolution-model estimate is one-order-of-magnitude smaller than the values in Fig. 4. We need to investigate more exotic hadronic mechanisms. At the same time, experimental efforts are needed to measure $b_{1}$ much accurately because there are only a few data points as shown in Fig. 3 with the large errors. However, it is fortunate that the JLab $b_{1}$ experiment was approved, and the measurement will start in a few years [8]. On the other hand, there are possibilities for investigating the antiquark tensor polarization by polarized Drell-Yan processes, such as $p+\vec{d} \rightarrow \mu^{+} \mu^{-}+X$ and $\pi+\vec{d} \rightarrow \mu^{+} \mu^{-}+X$, at hadron facilities, such as Fermilab-MI, RHIC, CERN-COMPASS, J-PARC, GSI-FAIR, and U70 [12]. For example, a tensor spin symmetry in $p \vec{d}$ Drell-Yan is given by

$$
A_{U Q_{0}}\left(\operatorname{large} x_{F}\right) \simeq \frac{\sum_{i} e_{i}^{2} \bar{q}_{i}\left(x_{1}\right) \delta_{T} q_{i}\left(x_{2}\right)}{\sum_{i} e_{i}^{2} \bar{q}_{i}\left(x_{1}\right) q_{i}\left(x_{2}\right)},
$$

so that $\delta_{T} q_{i}$ can be measured directly. It is similar to the case that $\bar{u} / \bar{d} \neq 1$ was clarified by the Drell-Yan experiment although it was suggested by the Gottfried-sum-rule violation [11].

\section{Acknowledgements}

This work was supported by Ministry of Education, Culture, Sports, Science and Technology (MEXT) KAKENHI Grant No. 25105010.

\section{References}

[1] D. F. Geesaman, K. Saito, and A. W. Thomas, Ann. Rev. Nucl. Part. Sci. 45, 337 (1995); L. Frankfurt, V. Gusey, and M. Strikman, Phys. Rept. 512, 255 (2012).

[2] See http://nuint.kek.jp/ for activities of neutrino-nucleon interaction collaboration at J-PARC branch of KEK theory center; Y. Hayato, M. Hirai, W. Horiuchi, H. Kamano, S. Kumano, T. Murata, S. Nakamura, K. Saito, M. Sakuda, and T. Sato, to be submitted for publication.

[3] M. Hirai, S. Kumano, and T. -H. Nagai, Phys. Rev. C 76, 065207 (2007).

[4] K. J. Eskola, H. Paukkunen, and C. A. Salgado, JHEP 04, 065 (2009); D. de Florian, R. Sassot, P. Zurita, and M. Stratmann, Phys. Rev. D 85, 074028 (2012); K. Kovarik et al., arXiv:1509.00792.

[5] I. Schienbein et al., Phys. Rev. D 77, 054013 (2008); D 80, 094004 (2009); K. Kovarik et al., Phys. Rev. Lett. 106, 122301 (2011).

[6] S. Kumano, J. Phys.: Conf. Series 543, 012001 (2014).

[7] A. Airapetian et al. (HERMES Collaboration), Phys. Rev. Lett. 95, 242001 (2005);

[8] Proposal to Jefferson Lab PAC-38, J.-P. Chen et al. (2011); K. Slifer, talk at the Tensor Polarized Solid Target Workshop, March 10-12, 2014, JLab, Newport News, USA.

[9] S. Kumano, Phys. Rev. D 82, 017501 (2010); T.-Y. Kimura and S. Kumano, Phys. Rev. D 78, 117505 (2008).

[10] F. E. Close and S. Kumano, Phys. Rev. D 42, 2377 (1990).

[11] S. Kumano, Phys. Rept. 303, 183 (1998); G. T. Garvey and J.-C. Peng, Prog. Part. Nucl. Phys. 47, 203 (2001); J.-C. Peng and J.-W. Qiu, Prog. Part. Nucl. Phys. 76, 43 (2014).

[12] X. Jiang and D. Keller, personal communications; S. Hino and S. Kumano, Phys. Rev. D 59, 094026 (1999); 60, 054018 (1999); S. Kumano and M. Miyama, Phys. Lett. B 479, 149 (2000). 